\title{
Fixed point theorems in locally convex algebras and applications to nonlinear integral equations
}

Le Khanh Hung*

\section{"Correspondence:}

lekhanhhungdhv@gmail.com Department of Mathematics, Vinh University, 182 Le Duan, Vinh,

Vietnam

\begin{abstract}
In this paper, we give a fixed point theorem in locally convex algebras and present applications to the existence problem for a class of nonlinear integral equations with unbounded deviations.
\end{abstract}

Keywords: fixed point theorems; locally convex algebras; Frechet algebras; contractive mappings; nonlinear integral equation; unbounded deviations

\section{Introduction}

The research of functional integral equations and differential equations is a main object of investigations of nonlinear functional analysis. These equations occur in physical, biological, and economic problems. The existence of solutions of them are commonly proved by suitable fixed point theorems. In this paper, we establish a new fixed point theorem for continuous mappings on locally convex algebras. These results arose out of our examination of a particular functional integral with unbounded deviations. We noticed in our study that the fixed point theorems developed in the Banach algebras (see [1-5] and the references given therein) were not always useful in establishing existence principles for the problems that we are interested in Section 4. More precisely, the well-known results in Banach algebras are not applicable to nonlinear integral equations with unbounded deviations.

\section{Preliminaries}

Before stating the main results, we give some useful definitions, preliminaries which will be used in the sequel.

Let $X$ be a uniform space. Then uniform topology on $X$ is generated by the family of uniform continuous pseudometrics on $X \times X$ (see [6]). In this paper, by $(X, \mathcal{P})$ we mean a Hausdorff uniform space whose uniformity is generated by a saturated family of pseudometrics $\mathcal{P}=\left\{d_{\alpha}(x, y): \alpha \in I\right\}$, where $I$ is an index set. Note that $(X, \mathcal{P})$ is Hausdorff if and only if $d_{\alpha}(x, y)=0$ for all $\alpha \in I$ implies $x=y$.

Definition 2.1 ([7]) Let $(X, \mathcal{P})$ be a Hausdorff uniform space.

(1) The sequence $\left\{x_{n}\right\} \subset X$ is Cauchy if $d_{\alpha}\left(x_{n}, x_{m}\right) \rightarrow 0$ as $m, n \rightarrow+\infty$ for every $\alpha \in I$.

\section{囪 Springer}


(2) $X$ is said to be sequentially complete if every Cauchy sequence $\left\{x_{n}\right\}$ in $X$ converges to $x \in X$.

Definition $2.2([7])$ Let $j: I \rightarrow I$ be an arbitrary mapping of the index set $I$ into itself. The iterations of $j$ can be defined inductively

$$
j^{0}(\alpha)=\alpha, \quad j^{k}(\alpha)=j\left(j^{k-1}(\alpha)\right), \quad k=1,2, \ldots
$$

Now, we introduce the two classes of functions which play crucial roles in the fixed point theory. Sometimes, they are called control functions.

Let $\Phi=\{\phi\}$ be a class of functions with the properties:

(i) $\phi:[0,+\infty) \rightarrow[0,+\infty)$ is monotone non-decreasing and continuous;

(ii) $0<\phi(t)<t$ for all $t>0$ and $\phi(0)=0$.

Let $\Psi=\{\psi\}$ be a class of functions with the properties:

(i) $\psi:[0,+\infty) \rightarrow[0,+\infty)$ is monotone non-decreasing and continuous;

(ii) $\psi(0)=0$.

We need the following fact for the class of functions $\Phi$. It may be not original.

Lemma 2.3 For any $\phi \in \Phi$, if $K$ is a bounded subset of $[0,+\infty)$ then there exists $k \in(0,1)$ such that

$$
\phi(t) \leq k t
$$

for all $t \in K$.

Proof Since the closure $\bar{K}$ of $K$ is compact and $\phi$ is continuous, we have

$$
\max _{t \in \bar{K}} \phi(t)=\phi\left(t_{0}\right)<t_{0}
$$

for some $t_{0} \in \bar{K}$. Suppose that there no $0<k<1$ such that $\phi(t) \leq k t$ for all $t \in K$. Then, for each $n=1,2, \ldots$ we can find $t_{n} \in K$ such that

$$
\phi\left(t_{n}\right) \geq\left(1-\frac{1}{n}\right) t_{n}
$$

Since $\bar{K}$ is compact, there exists a subsequence $\left(t_{n_{k}}\right)$ of the sequence $\left(t_{n}\right)$ such that $t_{n_{k}} \rightarrow$ $t \in \bar{K}$. Letting $n_{k} \rightarrow+\infty$ in (1), we arrive at $\phi(t) \geq t$ and this is a contradiction.

Definition 2.4 ([7]) Let $(X, \mathcal{P})$ be a uniform space and $\left\{\phi_{\alpha}\right\}_{\alpha \in I} \subset \Phi$ be a family of functions. A mapping $T: X \rightarrow X$ is said to be $\Phi$-contractive on $X$ if

$$
d_{\alpha}(T x, T y) \leq \phi_{\alpha}\left(d_{j(\alpha)}(x, y)\right)
$$

for all $x, y \in X, \alpha \in I$ and for some fixed mapping $j: I \rightarrow I$.

The following is due to Angelov [8]. 
Theorem $2.5([7,8])$ Let $(X, \mathcal{P})$ be a Hausdorff sequentially complete uniform space and $T: X \rightarrow X$ be a mapping. Suppose that

(1) $T$ is $\Phi$-contractive;

(2) for every $\alpha \in I$ there exists a function $\bar{\phi}_{\alpha} \in \Phi$ such that

$$
\sup \left\{\phi_{j^{n}(\alpha)}(t): n=0,1,2, \ldots\right\} \leq \bar{\phi}_{\alpha}(t)
$$

and $\frac{\bar{\phi}_{\alpha}(t)}{t}$ is monotone non-decreasing;

(3) there is $x_{0} \in X$ such that for every $\alpha \in I$ there exists $q(\alpha)>0$ such that the inequality $d_{j^{n}(\alpha)}\left(x_{0}, T x_{0}\right) \leq q(\alpha)$ is valid for all $n=0,1, \ldots$.

Then $T$ has at least one fixed point in $X$.

Angelov added the following properties of $X$ for the uniqueness of fixed point.

Definition 2.6 ([7]) A uniform space $(X, \mathcal{P})$ is said to be $j$-bounded if for every $\alpha \in I$ and $x, y \in X$ there exists $q=q(x, y, \alpha)$ such that

$$
d_{j^{n}(\alpha)}(x, y) \leq q(x, y, \alpha)<+\infty, \quad \text { for all } n \in \mathbb{N} \text {. }
$$

Theorem $2.7([7,8])$ Suppose that the conditions of Theorem 2.5 are fulfilled. If $X$ is $j$ bounded then $F$ has a unique fixed point.

Remark 2.8 If $E$ is a locally convex space with a saturated family of seminorms $\left\{p_{\alpha}\right\}_{\alpha \in I}$, then the associated family of pseudometrics $\left\{d_{\alpha}\right\}_{\alpha \in I}$ defined by $d_{\alpha}(x, y)=p_{\alpha}(x-y)$ for every $x, y \in E$ and $\alpha \in I$. The uniform topology, which is generated by this family of pseudometrics $\left\{d_{\alpha}\right\}_{\alpha \in I}$, coincides with the original topology of the space $E$. Therefore, as a corollary of Theorem 2.5, we obtain the fixed point theorems in locally convex spaces.

Let $X$ be a locally convex space and $T: X \rightarrow X$. Then $T$ is called a compact operator if $\overline{T(X)}$ is a compact subset of $X$. Again $T$ is called totally bounded if for any bounded set $S$ of $X, T(S)$ is a totally bounded set of $X$. Further, $T$ is called completely continuous if it is continuous and totally bounded. Note that every compact operator is totally bounded. The two notions are equivalent on a bounded set of $X$.

The following theorem is called the Tikhonov-Schauder fixed point theorem.

Theorem 2.9 ([9]) Let E be a Hausdorff locally convex space, $C$ a convex subset of $E$ and $F: C \rightarrow E$ a continuous mapping such that

$$
F(C) \subset A \subset C
$$

with A compact. Then $F$ has at least one fixed point.

Throughout this paper, we consider associative and commutative algebras over the field $\mathbb{K}$ of complex numbers or real numbers.

Definition $2.10([10,11])$ Let $E$ be an algebra over $\mathbb{K}$. $E$ is called a topological algebra if

(1) $E$ is a topological vector space;

(2) the multiplication in $E$ is continuous. 
We recall that a seminorm on the vector space $E$ is a real-valued function $p: E \rightarrow R$ such that the following conditions are satisfied:

(i) $p(x) \geq 0$, for every $x \in E$;

(ii) $p(x+y) \leq p(x)+p(y)$, for any $x, y \in E$;

(iii) $p(\lambda x)=|\lambda| p(x)$, for any $\lambda \in K$ and $x \in E$.

Definition $2.11([10,11])$ Let $E$ be a topological algebra.

(1) A seminorm $p: E \rightarrow \mathbb{R}$ is called submultiplicative if $p(x y) \leq p(x) p(y)$ for any elements $x, y$ in $E$.

(2) A set $U \subset E$ is called multiplicative if $U \cdot U \subset U$.

Definition $2.12([10,11])$ The topological algebra $E$ is called a locally multiplicatively convex algebra if $E$ has a local basis consisting of multiplicative and convex sets.

In this paper, a locally multiplicatively convex algebra is briefly called a locally convex algebra. The following remark is due to [10] and [11].

Remark $2.13([10,11])$ Let $E$ be a locally convex algebra. Then one can show that its topology is defined by a saturated family $\mathcal{P}=\left\{p_{\alpha}\right\}_{\alpha \in I}$ of submultiplicative seminorms.

Example 2.14 Let $X=C(\mathbb{R} ; \mathbb{R})$ be the algebra consisting all continuous functions from $R$ to $R$. Then $X$ is a locally convex algebra that defined by the countable family of submultiplicative seminorms

$$
p_{n}(x)=\max _{t \in[-n, n]}|x(t)|, \quad x \in X, n=1,2, \ldots
$$

For more basis material as regards the theory of locally convex algebra, we refer the reader to [10, 12], and [11].

\section{Fixed point theorems in locally convex algebras}

Let $X$ be a locally convex algebra with a saturated family of seminorms $\left\{p_{\alpha}\right\}_{\alpha \in I}$.

Definition 3.1 The mapping $T: X \rightarrow X$ is said to be $\mathcal{D}$-Lipschitzian with the family of functions $\left\{\psi_{\alpha}\right\}_{\alpha \in I}$ if

$$
p_{\alpha}(T x-T y) \leq \psi_{\alpha}\left(p_{j(\alpha)}(x-y)\right)
$$

for all $x, y \in X$ and $\alpha \in I$, where $\left\{\psi_{\alpha}\right\}_{\alpha \in I}$ is a subfamily of $\Psi$.

If $\psi_{\alpha}(t)=k_{\alpha} t$ for all $t \geq 0$, where $k_{\alpha}$ is a real number for all $\alpha \in I$, then $T$ is called Lipschitzian with the family of Lipschitz constants $\left\{k_{\alpha}\right\}_{\alpha \in I}$.

Theorem 3.2 Let $X$ be a locally convex algebra such that topology of $X$ is Hausdorff sequentially complete. Let $S$ be a closed, convex and bounded subset of $X$ and $A: X \rightarrow X$, $B: S \rightarrow X$ be two operators such that 
(A1) $A$ is $\mathcal{D}$-Lipschitzian with the family of functions $\left\{\psi_{\alpha}\right\}$;

(A2) $B$ is completely continuous and $x=A x B y$ implies $x \in S$ for every $y \in S$;

(A3) $p_{j(\alpha)}(x-y) \leq p_{\alpha}(x-y)$ for every $x, y \in S$ and $\alpha \in I$;

(A4) for every $x \in X$ and for every $\alpha \in I$, there exists $q(\alpha, x)$ such that

$$
p_{j^{k}(\alpha)}(x) \leq q(\alpha, x)<+\infty
$$

for all $k=0,1,2, \ldots$ In particular, $p_{j^{k}(\alpha)}(x) \leq q(\alpha)<+\infty$ for every $x \in S$ and for all $k=0,1,2, \ldots$;

(A5) for each $\alpha \in I$,

$$
M_{\alpha} \psi_{\alpha}(t)<t
$$

for all $t>0$ and there exists $\bar{\phi}_{\alpha} \in \Phi$ such that $\frac{\bar{\phi}_{\alpha}(t)}{t}$ is non-decreasing and

$$
\sup \left\{M_{j^{k}(\alpha)} \psi_{j^{k}(\alpha)}(t): k=0,1,2, \ldots\right\} \leq \bar{\phi}_{\alpha}(t)
$$

for every $t>0$, where $M_{\alpha}=\sup \left\{p_{\alpha}(B(x)): x \in S\right\}, \alpha \in I$.

Then the operator equation $x=A x B x$ has a solution.

Proof Now, let $y \in S$, we define a mapping $A_{y}: X \rightarrow X$ by

$$
A_{y}(x)=A x B y, \quad x \in X
$$

First, we claim that $A_{y}$ is $\mathcal{D}$-Lipschitzian. For each $\alpha \in I$, we have

$$
\begin{aligned}
p_{\alpha}\left(A_{y} x_{1}-A_{y} x_{2}\right) & =p_{\alpha}\left(A x_{1} B y-A x_{2} B y\right) \\
& \leq p_{\alpha}\left(A x_{1}-A x_{2}\right) p_{\alpha}(B y) \\
& \leq p_{\alpha}\left(A x_{1}-A x_{2}\right) M_{\alpha} \\
& \leq M_{\alpha} \psi_{\alpha}\left(p_{j(\alpha)}\left(x_{1}-x_{2}\right)\right) \\
& =\phi_{\alpha}\left(p_{j(\alpha)}\left(x_{1}-x_{2}\right)\right),
\end{aligned}
$$

where $\phi_{\alpha}=M_{\alpha} \psi_{\alpha} \in \Phi$. Then it is easy to see that all conditions of Theorem 2.7 are fulfilled for $A_{y}$ with every $\alpha \in I$. Hence, we get a unique fixed point $x^{*} \in X$ of $A_{y}$, that is,

$$
x^{*}=A_{y} x^{*}=A x^{*} B y .
$$

Since condition (A2) holds, we have $x^{*} \in S$. Now, we define a mapping $N: S \rightarrow X$ by

$$
N y=z
$$

where $z \in X$ is the unique solution of the equation

$$
z=A z B y, \quad z \in S
$$


Now, we show that $N$ is continuous. Let $\left\{y_{n}\right\}$ be a sequence in $S$ converging to a point $y$. Since $S$ is closed, we obtain $y \in S$. For each $\alpha \in I$, we have

$$
\begin{aligned}
p_{\alpha}\left(N y_{n}-N y\right) & =p_{\alpha}\left(A z_{n} B y_{n}-A z B y\right)=p_{\alpha}\left(A z_{n} B y_{n}-A z B y_{n}+A z B y_{n}-A z B y\right) \\
& \leq p_{\alpha}\left(\left(A z_{n}-A z\right) B y_{n}\right)+p_{\alpha}\left(A z\left(B y_{n}-B y\right)\right) \\
& \leq p_{\alpha}\left(A z_{n}-A z\right) p_{\alpha}\left(B y_{n}\right)+p_{\alpha}(A z) p_{\alpha}\left(B y_{n}-B y\right) .
\end{aligned}
$$

For each $n=1,2, \ldots$, since $A$ is $\mathcal{D}$-Lipschitzian, in view (2) and the condition (A3), we have

$$
\begin{aligned}
p_{\alpha}\left(A z_{n}-A z\right) p_{\alpha}\left(B y_{n}\right) & \leq \psi_{\alpha}\left(p_{j(\alpha)}\left(z_{n}-z\right)\right) M_{\alpha} \\
& =\phi_{\alpha}\left(p_{j(\alpha)}\left(z_{n}-z\right)\right)=\phi_{\alpha}\left(p_{j(\alpha)}\left(N y_{n}-N y\right)\right) \\
& \leq \phi_{\alpha}\left(p_{\alpha}\left(N y_{n}-N y\right)\right)
\end{aligned}
$$

Hence

$$
p_{\alpha}\left(N y_{n}-N y\right) \leq \phi_{\alpha}\left(p_{\alpha}\left(N y_{n}-N y\right)\right)+p_{\alpha}(A z) p_{\alpha}\left(B y_{n}-B y\right) .
$$

Letting $n \rightarrow+\infty$ and by the continuity of $B$, we get

$$
\begin{aligned}
\limsup _{n \rightarrow+\infty} p_{\alpha}\left(N y_{n}-N y\right) \leq & \limsup _{n \rightarrow+\infty} \phi_{\alpha}\left(p_{\alpha}\left(N y_{n}-N y\right)\right) \\
& +\limsup _{n \rightarrow+\infty} p_{\alpha}(A z) p_{\alpha}\left(B y_{n}-B y\right) \\
= & \limsup _{n \rightarrow+\infty} \phi_{\alpha}\left(p_{\alpha}\left(N y_{n}-N y\right)\right) \\
= & \phi_{\alpha}\left(\limsup _{n \rightarrow+\infty} p_{\alpha}\left(N y_{n}-N y\right)\right) .
\end{aligned}
$$

Since $S$ is bounded and $N(S) \subset S$ we have

$$
\limsup _{n \rightarrow+\infty} p_{\alpha}\left(N y_{n}-N y\right)=r<+\infty
$$

for each $\alpha \in I$. Therefore, it follows from (3) that

$$
r \leq \phi_{\alpha}(r)
$$

This implies that

$$
r=\limsup _{n \rightarrow+\infty} p_{\alpha}\left(N y_{n}-N y\right)=0
$$

for all $\alpha \in I$. Hence $\left\{N y_{n}\right\}$ converges to $N y$. This proves that $N$ is continuous.

Next we show that $N$ is a compact mapping. Since $S$ is bounded and $p_{j(\alpha)}$ is continuous, $p_{j(\alpha)}(S)$ is a bounded subset of $[0,+\infty)$. Applying Lemma 2.3 , we can seek $k_{\alpha} \in[0,1)$ such that

$$
\phi_{\alpha}\left(p_{j(\alpha)}(x-y)\right)<k_{\alpha} p_{\alpha}(x-y)
$$


for all $x, y \in S$. Now, for each $\alpha \in I$ and for any $z \in S$ we have

$$
p_{\alpha}(A z) \leq p_{\alpha}(A a)+p_{\alpha}(A z-A a) \leq p_{\alpha}(A a)+k_{\alpha} p_{\alpha}(z-a) \leq c_{\alpha},
$$

where $c_{\alpha}=p_{\alpha}(A a)+2 q(\alpha)$, and $a \in S$.

Let $U$ be an open neighborhood of 0 in $X$. Without loss of generality, we may assume that

$$
U=\left\{x \in X: p_{\alpha_{1}}(x)<\varepsilon, p_{\alpha_{2}}(x)<\varepsilon, \ldots, p_{\alpha_{m}}(x)<\varepsilon\right\}
$$

for some $\varepsilon>0, \alpha_{1}, \ldots, \alpha_{m} \in I$. Since $B$ is completely continuous, $B(S)$ is totally bounded. Then there is a finite set $Y=\left\{y_{1}, \ldots, y_{n}\right\} \subset S$ such that

$$
B(S) \subset \bigcup_{l=1}^{n} B\left(y_{l}\right)+V,
$$

where

$$
\begin{aligned}
V= & \left\{x \in X: p_{\alpha_{1}}(x)<\left(\frac{1-k_{\alpha_{1}}}{c_{\alpha_{1}}}\right) \varepsilon, \ldots, p_{\alpha_{m}}(x)<\left(\frac{1-k_{\alpha_{n}}}{c_{\alpha_{n}}}\right) \varepsilon,\right. \\
& \left.p_{j\left(\alpha_{1}\right)}(x)<\varepsilon, \ldots, p_{j\left(\alpha_{m}\right)}(x)<\varepsilon\right\} .
\end{aligned}
$$

Therefore, for any $y \in S$ we have $y_{l} \in Y$ such that

$$
p_{\alpha_{i}}\left(B y-B y_{l}\right)<\left(\frac{1-k_{\alpha_{i}}}{c_{\alpha_{i}}}\right) \varepsilon, \quad p_{j\left(\alpha_{i}\right)}\left(B y-B y_{l}\right)<\varepsilon
$$

for every $i=1,2, \ldots, m$. Also, for every $i=1,2, \ldots, m$ and $z_{l}=N y_{l}$, we have

$$
\begin{aligned}
p_{\alpha_{i}}\left(z-z_{l}\right) & =p_{\alpha_{i}}\left(N y-N y_{l}\right)=p_{\alpha_{i}}\left(A z B y-A z_{l} B y_{l}\right) \\
& \leq p_{\alpha_{i}}\left(A z B y-A z_{l} B y\right)+p_{\alpha_{i}}\left(A z_{l} B y-A z_{l} B y_{l}\right) \\
& \leq p_{\alpha_{i}}\left(A z-A z_{l}\right) p_{\alpha_{i}}(B y)+p_{\alpha_{i}}\left(A z_{l}\right) p_{\alpha_{i}}\left(B y-B y_{l}\right) \\
& <\phi_{\alpha_{i}}\left(p_{j\left(\alpha_{i}\right)}\left(z-z_{l}\right)\right)+c_{\alpha_{i}}\left(\frac{1-k_{\alpha_{i}}}{c_{\alpha_{i}}}\right) \varepsilon \\
& <k_{\alpha_{i}} p_{j\left(\alpha_{i}\right)}\left(z-z_{l}\right)+\left(1-k_{\alpha_{i}}\right) \varepsilon \\
& \leq k_{\alpha_{i}} p_{\alpha_{i}}\left(z-z_{l}\right)+\left(1-k_{\alpha_{i}}\right) \varepsilon .
\end{aligned}
$$

This yields

$$
p_{\alpha_{i}}\left(z-z_{l}\right)<\varepsilon
$$

for every $l=1,2, \ldots, n$. Hence $N y-N y_{l}=z-z_{l} \in U$, that is, $z=N y \in z_{l}+U$. It follows that

$$
N(S) \subset \bigcup_{l=1}^{n}\left(z_{l}+U\right) .
$$


This implies that $N$ is totally bounded. Combining this fact and the continuity of $N$, we can conclude that $N$ is a compact operator.

Since $S$ is convex, closed, and bounded, applying Tikhonov-Schauder's fixed theorem, we find that $N$ has a fixed point in $S$. Then by the definition of $N$, we obtain

$$
x=N x=A(N x) B x=A x B x .
$$

This proves that the operator equation $x=A x B x$ has a solution in $S$. The theorem is proved.

Remark 3.3 If $S$ contains 0 then the condition (A3) implies condition (A4). Indeed, since $0 \in S$ and condition (A3) we have

$$
p_{j(\alpha)}(x) \leq p_{\alpha}(x)
$$

for every $x \in X$ and $\alpha \in I$. It follows that

$$
p_{j^{k}(\alpha)}(x) \leq p_{\alpha}(x)
$$

for every $x \in X$ and $k=1,2, \ldots$ Moreover, since $S$ is bounded, for every $\alpha \in I$, there exists $q(\alpha)$ such that

$$
p_{j^{k}(\alpha)}(x) \leq q(\alpha)
$$

for all $x \in S$.

We can immediately obtain the following corollary.

Corollary 3.4 Let $X$ be a locally convex algebra such that the topology of $X$ is Hausdorff sequentially complete. Let $S$ be a closed, convex, and bounded subset of $X$ and let $A: X \rightarrow X$, $B: S \rightarrow X$ be two operators such that:

(B1) $A$ is Lipschitzian with the family of Lipschitz constants $\left\{k_{\alpha}\right\}$;

(B2) $B$ is completely continuous and $x=A x B y$ implies $x \in S$ for every $y \in S$;

(B3) $p_{j(\alpha)}(x-y) \leq p_{\alpha}(x-y)$ for every $x, y \in S$ and $\alpha \in I$;

(B4) for every $x \in X$ and for every $\alpha \in I$, there exists $q(\alpha, x)$ such that

$$
p_{j^{k}(\alpha)}(x) \leq q(\alpha, x)<+\infty
$$

for all $k=0,1,2, \ldots$. In particular, $p_{j^{k}(\alpha)}(x) \leq q(\alpha)<+\infty$ for every $x \in S$ and for all $k=0,1,2, \ldots$;

(B5) for each $\alpha \in I$,

$$
M_{\alpha} k_{\alpha}<1
$$

and

$$
\sup \left\{M_{j^{k}(\alpha)} k_{j^{k}(\alpha)}: k=0,1,2, \ldots\right\} \leq r_{\alpha}<1,
$$

where $M_{\alpha}=\sup \left\{p_{\alpha}(B(x)): x \in S\right\}, \alpha \in I$.

Then the operator equation $x=A x B x$ has a solution. 


\section{Applications to nonlinear integral equations}

In this section, we apply the previous result to investigate the existence of a solution to nonlinear integral equations with unbounded deviations. Let us consider the following integral equation:

$$
\begin{aligned}
x(t)= & F\left(t, \int_{0}^{\Delta_{1}(t)} x(s) d s, \ldots, \int_{0}^{\Delta_{m}(t)} x(s) d s, x\left(\tau_{1}(t)\right), \ldots, x\left(\tau_{n}(t)\right)\right) \\
& \times\left[q(t)+\int_{0}^{t} f(s, x(s)) d s\right]
\end{aligned}
$$

for $t>0$, where we have an unknown function $x(t)$, the deviations $\Delta_{i}, \tau_{j}:[0,+\infty) \rightarrow$ $[0,+\infty)$ are continuous functions, in the general case, unbounded, and $q:[0,+\infty) \rightarrow \mathbb{R}$, $f:[0,+\infty) \times \mathbb{R} \rightarrow \mathbb{R}$ are continuous functions. Note that, since the deviations are unbounded, we cannot apply the well-known fixed point theorems in Banach algebras (see [2-5], and the references given therein) for the above integral equations.

By a solution of the FIE (4), we mean a continuous function $x:[0,+\infty) \rightarrow \mathbb{R}$ that satisfies FIE (4) on $\mathbb{R}_{+}:=[0,+\infty)$. Let $X=C\left(\mathbb{R}_{+}, \mathbb{R}\right)$ be the locally convex algebra (in fact, Frechet algebra) of all continuous real-valued functions on $R_{+}$with a family of seminorms,

$$
p_{[0, n]}(x)=\max \{|x(t)|: t \in[0, n]\} .
$$

As in [7] and [2], we shall adopt the following assumptions.

\section{Assumption 4.1}

(C1) The functions $\Delta_{i}(t): \mathbb{R}_{+} \rightarrow \mathbb{R}_{+}, i=1,2, \ldots, m ; \tau_{l}(t): \mathbb{R}_{+} \rightarrow \mathbb{R}_{+}, l=1,2, \ldots, n$, are continuous and $\Delta_{i}(t) \leq t, \tau_{l}(t) \leq t$ for every $t>0$.

(C2) The function $F:\left(t, u_{1}, u_{2}, \ldots, u_{m}, v_{1}, \ldots, v_{n}\right): \mathbb{R}_{+} \times \mathbb{R}^{m+n} \rightarrow[0,1]$ is continuous and satisfies the conditions

$$
\begin{aligned}
& \left|F\left(t, u_{1}, \ldots, u_{m}, v_{1}, \ldots, v_{n}\right)-F\left(t, \bar{u}_{1}, \ldots, \bar{u}_{m}, \bar{v}_{1}, \ldots, \bar{v}_{n}\right)\right| \\
& \quad \leq \Omega\left(t,\left|u_{1}-\bar{u}_{1}\right|, \ldots,\left|u_{m}-\bar{u}_{m}\right|,\left|v_{1}-\bar{v}_{1}\right|, \ldots,\left|v_{n}-\bar{v}_{n}\right|\right),
\end{aligned}
$$

where the function $\Omega\left(t, x_{1}, \ldots, x_{m}, y_{1}, \ldots, y_{n}\right): \mathbb{R}_{+}^{m+n+1} \rightarrow \mathbb{R}_{+}$is continuous in $t$, non-decreasing and continuous in each $x_{i}, y_{l}, \Omega(t, a y, \ldots, a y, y, \ldots, y)<y$ for every constant $a>0$ and $\frac{\Omega(t, a y, \ldots, a y, y, \ldots, y)}{y}$ is non-decreasing in $y$.

(C3) $q$ is uniformly continuous on $\mathbb{R}_{+},\|q\|_{\infty}=\sup _{t \in \mathbb{R}_{+}}|q(t)|<1$ and $\int_{0}^{+\infty}|f(s, x(s))| d s<1-\|q\|_{\infty}$ for every $x \in C\left(\mathbb{R}_{+}, \mathbb{R}\right)$ with $|x(t)| \leq 1$ for all $t$.

Theorem 4.2 Under assumptions (C1), (C2), and (C3), (4) has at least one solution $x=x(t)$ which belongs to the space $C\left(\mathbb{R}_{+}, \mathbb{R}\right)$.

Proof Let $S=\left\{x \in C\left(\mathbb{R}_{+}, \mathbb{R}\right): p_{[0, n]}(x) \leq 1, n=1,2, \ldots\right\}$. It is easy to see that $S$ is a convex, closed, and bounded subset of $C\left(\mathbb{R}_{+}, \mathbb{R}\right)$. Let us consider two operators $A, B$ defined on $C\left(\mathbb{R}_{+}, \mathbb{R}\right)$ by

$$
(A x)(t)=F\left(t, \int_{0}^{\Delta_{1}(t)} x(s) d s, \ldots, \int_{0}^{\Delta_{m}(t)} x(s) d s, x\left(\tau_{1}(t)\right), \ldots, x\left(\tau_{n}(t)\right)\right)
$$


and

$$
(B x)(t)=q(t)+\int_{0}^{t} f(s, x(s)) d s,
$$

for all $t \in R_{+}$.

Then (4) is equivalent to the operator equation

$$
x=A x B x
$$

on $C\left(\mathbb{R}_{+}, \mathbb{R}\right)$. Now, we claim that the operators $A$ and $B$ satisfy all conditions of Theorem 3.2 with $S$ as previously mentioned.

Now, we consider the index set $I=\{[0, k]: k=1,2, \ldots\}$ and for each $[0, k] \in I$, we set

$$
r(k):=\left[\max \left\{\max _{t \in[0, k]} \Delta_{i}(t), \max _{t \in[0, k]} \tau_{l}(t): i=1,2, \ldots, m ; l=1,2, \ldots, n\right\}\right],
$$

where $[x]$ is the integer part of $x \in \mathbb{R}$. By the condition (C1) we get $r(k) \leq k$. Let us

$$
N(k)= \begin{cases}r(k)+1 & \text { if } r(k)<k, \\ r(k) & \text { if } r(k)=k .\end{cases}
$$

We define a map $j: I \rightarrow I$ by

$$
j([0, k])=[0, N(k)] .
$$

It is easy to see that

$$
\left[0, \max _{t \in[0, k]} \Delta_{i}(t)\right] \subset j([0, k]) \text { and } \quad\left[0, \max _{t \in[0, k]} \tau_{l}(t)\right] \subset j([0, k])
$$

for all $i=1,2, \ldots, m, l=1,2, \ldots, n$.

For each $k=1,2, \ldots$, we put $\Delta_{k}^{i}=\max _{t \in[0, k]} \Delta_{i}(t)$ and

$$
a_{k}=\max \left\{\Delta_{k}^{i}: i=1,2, \ldots, m\right\} .
$$

It follows from $(\mathrm{C} 2)$ that the function

$$
\phi_{[0, k]}(y):=\sup \left\{\Omega\left(t, a_{k} y, \ldots, a_{k} y, y, \ldots, y\right): t \in[0, k]\right\}
$$

is continuous and non-decreasing, $\phi_{[0, k]}(y)<y$ for every $y>0$, and $\frac{\phi_{[0, k]}(y)}{y}$ is nondecreasing.

Next, we shall show that $A$ is $\mathcal{D}$-Lipschitzian. Indeed, for each $[0, n] \in I$ and for every $x, y \in C\left(\mathbb{R}_{+}, \mathbb{R}\right)$, using $(\mathrm{C} 2)$, we have

$$
\begin{aligned}
& p_{[0, k]}(A x-A y) \\
& \quad=\sup _{t \in[0, k]}|(A x)(t)-(A y)(t)|
\end{aligned}
$$




$$
\begin{aligned}
= & \sup _{t \in[0, k]} \mid F\left(t, \int_{0}^{\Delta_{1}(t)} x(s) d s, \ldots, \int_{0}^{\Delta_{m}(t)} x(s) d s, x\left(\tau_{1}(t)\right), \ldots, x\left(\tau_{n}(t)\right)\right) \\
& -F\left(t, \int_{0}^{\Delta_{1}(t)} y(s) d s, \ldots, \int_{0}^{\Delta_{m}(t)} y(s) d s, y\left(\tau_{1}(t)\right), \ldots, y\left(\tau_{n}(t)\right)\right) \mid \\
\leq & \sup _{t \in[0, k]} \Omega\left(t,\left|\int_{0}^{\Delta_{1}(t)}(x(s)-y(s)) d s\right|, \ldots,\left|\int_{0}^{\Delta_{m}(t)}(x(s)-y(s)) d s\right|,\right. \\
& \left.\left|x\left(\tau_{1}(t)\right)-y\left(\tau_{1}(t)\right)\right|, \ldots,\left|x\left(\tau_{n}(t)\right)-y\left(\tau_{n}(t)\right)\right|\right) \\
\leq & \sup _{t \in[0, k]} \Omega\left(t, \int_{0}^{\Delta_{1}(t)}|x(s)-y(s)| d s, \ldots, \int_{0}^{\Delta_{m}(t)}|x(s)-y(s)| d s,\right. \\
& \left.\left|x\left(\tau_{1}(t)\right)-y\left(\tau_{1}(t)\right)\right|, \ldots,\left|x\left(\tau_{n}(t)\right)-y\left(\tau_{n}(t)\right)\right|\right) .
\end{aligned}
$$

For each $t \in[0, k]$, in view to (5), we have

$$
\begin{aligned}
\Omega(t, & \int_{0}^{\Delta_{1}(t)}|x(s)-y(s)| d s, \ldots, \int_{0}^{\Delta_{m}(t)}|x(s)-y(s)| d s, \\
& \left.\left|x\left(\tau_{1}(t)\right)-y\left(\tau_{1}(t)\right)\right|, \ldots,\left|x\left(\tau_{n}(t)\right)-y\left(\tau_{n}(t)\right)\right|\right) \\
\leq & \Omega\left(t,\left|\Delta_{1}(t)\right| \max _{s \in\left[0, \max _{t \in[0, k]} \Delta_{1}(t)\right]}|x(s)-y(s)|, \ldots,\right. \\
& \left|\Delta_{m}(t)\right| \max _{s \in\left[0, \max _{t \in[0, k]} \Delta_{m}(t)\right]}|x(s)-y(s)|, \\
& \left.\max _{s \in\left[0, \max _{t \in[0, k]} \tau_{1}(t)\right]}|x(s)-y(s)|, \ldots, \max _{s \in\left[0, \max _{t \in[0, k]} \tau_{n}(t)\right]}|x(s)-y(s)|\right) \\
\leq & \Omega\left(t,\left|\Delta_{1}(t)\right| \max _{s \in j([0, k])}|x(s)-y(s)|, \ldots,\left|\Delta_{m}(t)\right| \max _{s \in j([0, k])}|x(s)-y(s)|,\right. \\
& \left.\max _{s \in j([0, k])}|x(s)-y(s)|, \ldots, \max _{s \in j([0, k])}|x(s)-y(s)|\right) \\
\leq & \Omega\left(t, \Delta_{k}^{1} p_{j([0, k])}(x-y), \ldots, \Delta_{k}^{m} p_{j([0, k])}(x-y),\right. \\
& \left.p_{j([0, k])}(x-y), \ldots, p_{j([0, k])}(x-y)\right) \\
\leq & \Omega\left(t, a_{k} p_{j([0, k])}(x-y), \ldots, a_{k} p_{j([0, k])}(x-y),\right. \\
& \left.p_{j([0, k])}(x-y), \ldots, p_{j([0, k])}(x-y)\right) .
\end{aligned}
$$

From (6) and (7), we obtain

$$
\begin{aligned}
p_{[0, k]}(A x-A y) \\
\leq \sup _{t \in[0, k]} \Omega\left(t, a_{k} p_{j([0, k])}(x-y), \ldots, a_{k} p_{j([0, k])}(x-y),\right. \\
\left.\quad p_{j([0, k])}(x-y), \ldots, p_{j([0, k])}(x-y)\right) \\
=\phi_{[0, k]}\left(p_{j([0, k])}(x-y)\right) .
\end{aligned}
$$

This proves that $A$ is $\mathcal{D}$-Lipschitzian. 
Moreover, by assumption (C1) and the definition of $j$ we have

$$
j^{k}([0, n]) \subset[0, n]
$$

for all $n$ and $k$. It follows that the conditions (A3) and (A4) of Theorem 3.2 are satisfied. By the above reasoning, we have

$$
\phi_{j k([0, n])}(y) \leq \phi_{[0, n]}(y):=\bar{\phi}_{[0, n]}(y) \in \Phi .
$$

Next, we shall deal with some estimations on

$$
M_{[0, n]}:=\sup \left\{p_{[0, n]}(B(x)): x \in S\right\} .
$$

We have

$$
\begin{aligned}
p_{[0, n]}(B(x)) & =\sup _{t \in[0, n]}\left|q(t)+\int_{0}^{t} f(s, x(s)) d s\right| \\
& \leq \sup _{t \in[0, n]}|q(t)|+\sup _{t \in[0, n]} \int_{0}^{t}|f(s, x(s))| d s \\
& \leq\|q\|_{\infty}+\int_{0}^{+\infty}|f(s, x(s))| d s \\
& <\|q\|_{\infty}+\left(1-\|q\|_{\infty}\right)=1
\end{aligned}
$$

for every $n=1,2, \ldots$ and $x \in S$. Hence $M_{[0, n]} \leq 1$, so that $M_{[0, n]} \phi_{[0, n]}(t)<t$ for every $t>0$ and the condition (A5) of Theorem 3.2 holds.

Now, we shall check the condition (A2). Firstly, we show that $B$ is completely continuous. Suppose $\left(x_{k}\right) \subset S$ and $x_{k} \rightarrow x$. Since $S$ is closed, we have $x \in S$. By the definition of seminorms $p_{[0, n]}$, we can deduce that $\left(x_{k}\right)$ uniformly convergent to $x$ on $[0, n]$ for each $n=1,2, \ldots$. It follows that $\left|f\left(s, x_{k}(s)\right)-f(s, x(s))\right| \rightarrow 0$ as $k \rightarrow+\infty$ for every $s \in[0,+\infty)$. Moreover, by the condition (C3), we infer the $g(s):=f(s, x(s))$ is a bounded function on $[0, n]$ for each $n=1,2, \ldots$.

For each $k=1,2, \ldots$, set $g_{k}(s)=f\left(s, x_{k}(s)\right)$, for every $s \in[0, n]$. We show that $\left(g_{k}\right)$ be a bounded sequence on $[0, n]$. Suppose to the contrary, for each $k=1,2, \ldots$ there exists $s_{k} \in$ $[0, n]$ such that

$$
\left|g_{k}\left(s_{k}\right)\right|=\left|f\left(s_{k}, x_{k}\left(s_{k}\right)\right)\right|>k
$$

Since $[0, n]$ is compact, there exists a subsequence $\left(s_{k_{j}}\right)$ of $\left(s_{k}\right)$ which converges to $s_{0} \in$ $[0, n]$. Since $\left\{x_{k}\right\}$ is uniformly convergent to $x$ on $[0, n]$, we have $x_{k_{j}}\left(s_{k_{j}}\right) \rightarrow x\left(s_{0}\right)$ as $k_{j} \rightarrow \infty$. Therefore, let $k_{j} \rightarrow \infty$ in (9) and using continuity of $f$ we infer $\left|f\left(s_{0}, x\left(s_{0}\right)\right)\right|=\infty$. Hence, $f$ is unbounded on $[0, n]$. This is a contradiction.

Applying the Lebesgue dominated convergence theorem, we have

$$
\int_{0}^{n}\left|f\left(s, x_{k}(s)\right)-f(s, x(s))\right| d s \rightarrow 0
$$


as $k \rightarrow \infty$. From

$$
\begin{aligned}
p_{[0, n]}\left(B\left(x_{k}\right)-B(x)\right) & =\max _{t \in[0, n]}\left|\int_{0}^{t} f\left(s, x_{k}(s)\right) d s-\int_{0}^{t} f(s, x(s)) d s\right| \\
& \leq \max _{t \in[0, n]} \int_{0}^{t}\left|f\left(s, x_{k}(s)\right)-f(s, x(s))\right| d s \\
& \leq \int_{0}^{n}\left|f\left(s, x_{k}(s)\right)-f(s, x(s))\right| d s,
\end{aligned}
$$

we conclude that

$$
p_{[0, n]}\left(B\left(x_{k}\right)-B(x)\right) \rightarrow 0
$$

as $k \rightarrow \infty$, for $n=1,2, \ldots$. This proves that $B$ is continuous.

Now, we show that $B(S)$ is totally bounded. It follows from (8) that $B(S) \subset S$. For any $\varepsilon>0$ and $B x \in B(S)$, since $\int_{0}^{+\infty}|f(s, x(s))| d s<1-\|q\|_{\infty}$ and $f(s, x(s))$ is continuous, it follows that $|f(s, x(s))| \leq M<+\infty$ for all $s \geq 0$, for some $M$. By the uniform continuity of $q$, we can seek the $\delta(\varepsilon)$ such that

$$
|q(t)-q(\tau)|<\frac{\varepsilon}{2}
$$

for all $|t-\tau|<\delta(\epsilon)$. Now, if we choose $\delta=\min \left\{\frac{\varepsilon}{2 M}, \delta(\varepsilon)\right\}$ then

$$
\begin{aligned}
|B x(t)-B x(\tau)| & =\left|q(t)-q(\tau)+\int_{0}^{t} f(s, x(s)) d s-\int_{0}^{\tau} f(s, x(s)) d s\right| \\
& \leq|q(t)-q(\tau)|+\int_{t}^{\tau}|f(s, x(s))| d s \\
& \leq \frac{\varepsilon}{2}+M|t-\tau|<\varepsilon
\end{aligned}
$$

for every $t, \tau \in[0,+\infty)$ and $|t-\tau|<\delta$. Hence $B(S)$ is a equicontinuous family. By ArzelaAscoli's theorem, we deduce that $B(S)$ is a precompact set of $C\left(\mathbb{R}_{+}, \mathbb{R}\right)$. Therefore, $B$ is completely continuous. It follows from condition $(\mathrm{C} 2)$ and condition $(\mathrm{C} 3)$ that the remain of condition (A2) of Theorem 3.2 is satisfied.

Finally, applying Theorem 3.2, we can conclude that (4) has a solution.

The following example is an illustration of Theorem 4.2.

Example 4.3 Consider the following nonlinear functional integral equation:

$$
x(t)=\frac{1}{2+|x(\tau(t))|}\left(t e^{-t^{2}}+\int_{0}^{t} s e^{-s^{2}\left(1+x^{2}(s)\right)} d s\right),
$$

where $\tau(t)$ is a continuous function on $[0,+\infty)$ and $\tau(t) \leq t$ for all $t \in[0,+\infty)$.

We will show that the equation has a solution on $C\left(\mathbb{R}^{+}, \mathbb{R}\right)$.

Set $q(t):=t e^{-t^{2}}$ and $f(s, x(s)):=s e^{-s^{2}\left(1+x^{2}(s)\right)}$. Since $q$ is continuous on $[0,+\infty)$ and $\lim _{t \rightarrow+\infty} q(t)=0$, it is easy to show that $q$ is uniform continuous. We also have

$$
\|q\|_{\infty}=\frac{1}{\sqrt{2 e}}<1
$$


and

$$
\int_{0}^{+\infty} s e^{-s^{2}\left(1+x^{2}(s)\right)} d s \leq \int_{0}^{+\infty} s e^{-s^{2}} d s=\frac{1}{2}<1-\frac{1}{\sqrt{2 e}}=1-\|q\|_{\infty}
$$

for every $x \in C\left(\mathbb{R}_{+}, \mathbb{R}\right)$.

Consider the function $F: \mathbb{R}_{+} \times \mathbb{R}^{2} \rightarrow[0,1]$ defined by

$$
F(t, u, v)=\frac{1}{2+|v|}
$$

for all $(t, u, v) \in \mathbb{R}_{+} \times \mathbb{R}^{2}$. It is easy to check that $F$ satisfies the condition (C2) with $\Omega(t, x, y)=\frac{y}{2}$. Hence, the Assumption 4.1 holds for the above $q, f$, and $F$.

\section{Competing interests}

The author declares that he has no competing interests.

\section{Author's contributions}

The author completed the paper himself. The author read and approved the final manuscript.

\section{Acknowledgements}

The author would like to thank the referee for very deep and useful comments, and his/her suggestions that contributed to the improvement of the manuscript. My thanks go to Professor Tran Van An and Kieu Phuong Chi for their valuable suggestions.

Received: 9 January 2015 Accepted: 16 April 2015 Published online: 29 April 2015

\section{References}

1. Banas, J, Taoudi, MA: Fixed points and solutions of operator equations for weak topology in Banach algebras. Taiwan. J. Math. (2014). doi:10.11650/tim.18.2014.3860

2. Dhage, BC: On a fixed point theorem in Banach algebras with applications. Appl. Math. Lett. 18(3), 273-280 (2005)

3. Dhage, BC: A fixed point theorem in Banach algebras involving three operators with applications. Kyungpook Math. J. 44(1), 145-155 (2004)

4. Dhage, BC, O'Regan, D: A fixed point theorem in Banach algebras with applications to functional integral equations. Funct. Differ. Equ. 7(3-4), 259-267 (2000)

5. Pathak, HK, Deepmala: Remarks on some fixed point theorems of Dhage. Appl. Math. Lett. 25(11), 1969-1975 (2012)

6. Kelley, JL: General Topology. Graduate Texts in Mathematics, vol. 27. Springer, New York (1975)

7. Angelov, VG: Fixed Points in Uniform Spaces and Applications. Cluj University Press, Cluj-Napoca (2009)

8. Angelov, VG: Fixed point theorem in uniform spaces and applications. Czechoslov. Math. J. 37(1), 19-33 (1987)

9. Agarwal, R, Meehan, M, O'Regan, D: Fixed Point Theory and Applications. Cambridge University Press, Cambridge (2004)

10. Beckenstein, E, Narici, L, Suffel, C: Topological Algebras. North-Holland Mathematics Studies. North-Holland, Amsterdam (1977)

11. Mallios, M: Topological Algebras: Selected Topics. North-Holland Mathematics Studies. North-Holland, Amsterdam (1986)

12. Goldmann, H: Uniform Frechet Algebras. North-Holland Mathematics Studies, vol. 162. North-Holland, Amsterdam (1990)

\section{Submit your manuscript to a SpringerOpen ${ }^{\circ}$ journal and benefit from:}

- Convenient online submission

- Rigorous peer review

- Immediate publication on acceptance

- Open access: articles freely available online

- High visibility within the field

- Retaining the copyright to your article 